\title{
Shapley and Roth Awarded Nobel Prize in Economics
}

LLOYD S. SHAPLEY, professor emeritus of economics and mathematics at the University of California, Los Angeles, and Alvin E. Roth, George Gund Professor of Economics and Business Administration at Harvard University and Harvard Business School, have been awarded the 2012 Nobel Prize in Economics "for the theory of stable allocations and the practice of market design." Though they worked independently of each other, the combination of Shapley's basic theory and Roth's empirical investigations, experiments, and practical design has generated a flourishing field of research and improved the performance of many markets.

\section{On the Work of Roth and Shapley}

The Notices asked Robert Aumann of the Hebrew University of Jerusalem to comment on the work of Alvin Roth and Lloyd Shapley. Aumann supplied the following description of their work.

"Matching markets" are the means by which we choose the many things in life that also choose usschools, universities, jobs, life partners, and so on. "Market design" is about designing these markets efficiently, in much the same sense that the price mechanism is efficient in ordinary markets. This is a highly nontrivial task. The fundamental theory of market design was laid out by Lloyd Shapley, Herbert Scarf, and the late David Gale in the sixties and seventies of the last century. It has been applied in practice, with resounding success, largely through the efforts of one individual: Alvin Roth.

In 1962 Shapley and Gale published a paper entitled "College admissions and the stability of marriage". There they developed an algorithm for matching-e.g., of men to women-that is "stable" in a very natural sense: No man and woman who are not matched to each other prefer each other to the partners with whom they are matched. In the early 1980s, Roth made a discovery with

DOI: http://dx.doi.org/10.1090/noti946 far-reaching consequences: In the first half of the twentieth century, the U.S. market for assigning medical students to hospitals (for the purpose of doing their residencies) had been in continuous turmoil. In the early 1950s, the American Hospital Association (AHA) introduced a computer algorithm for making these assignments, whereupon the previously chaotic market immediately became orderly. And Roth showed that the AHA algorithm was precisely the same as that of Gale and Shapley, though neither the AHA nor the inventors of their algorithm were familiar with the relevant theory.

This discovery opened the door to the application of the Gale-Shapley algorithm to a wide variety of important matching markets: in addition to the medical programs, perhaps most importantly to very large-scale programs of assigning students to schools, programs that are in actual use in New York and Boston and are under development in Denver and New Orleans. It also led to a large literature that modifies the algorithm to take account of new conditions that were not previously envisaged (e.g., when two medical students are married to each other and require residencies in the same hospital or at least in the same town). In addition to his applied work, Roth is a leader in these later theoretical developments.

Another very important development in market design is based on a paper entitled "On cores and indivisibility", published by Shapley and Scarf in 1974. This paper lays out the theory of discrete exchange markets: markets where large indivisible items-like houses-are exchanged without any money changing hands. While initially the assumption of exchange without money appeared to be of largely theoretical interest, Roth brilliantly used the Shapley-Scarf results to design practical methods for facilitating donations of kidneys from potential donors to recipients, where the recipient 
intended by the original donor is unable to receive the kidney because of medical incompatibility.

Market design enables highly practical applications of sophisticated and deep theoretical results. Unlike much of economic theory, this really works in the real world. In my opinion, the work of Shapley and Roth in market design is the most outstanding success story in game theory and economics, more so even than auction theory, which has enjoyed great success and for which William Vickrey was rightfully awarded the 1996 Bank of Sweden Prize in Economic Sciences in Honor of Alfred Nobel.

In addition to the work just described, which laid the basis for market design, Shapley has been the top game theorist in the world for some sixty years-ever since the early fifties of the last century. He has been at the forefront of just about every major development in game theory. Perhaps his most visible impact has been on the "cooperative" or coalitional side of the theory, but also on the "noncooperative" or strategic side, his work has been of major importance. Here we mention only some of the outstanding items, starting with the coalitional side.

1. Development of the core as an independent solution concept for coalitional games in the early fifties. This led to several developments of major importance, as outlined below (items 2 and 3).

2 . The principle of equivalence between the core of a large market and its competitive outcomes, as developed by Shapley and Shubik, Debreu, Scarf, and subsequently by many scores of others. For the first time, this work provided a firm theoretical foundation for the notion of competition-the "invisible hand" of Adam Smith. It is undoubtedly the most fundamental and significant application ever of game theory to economic theory.

3. The development of the notion of games with a continuum of players - "oceanic games"-by Shapley and Fields Medal winner John Milnor in the early sixties. The model of oceanic games turned out to have an enormous impact, including most prominently on the equivalence principle (item 2 above), but also on many other areas, including oligopoly, cost allocation, and even the routing of traffic.

4. Development of the "Shapley value"-an a priori evaluation of the "expected payoff" or "strength" of a player in a coalitional game. On this concept, I wrote in 1985 as follows: "The Shapley value is perhaps the most 'successful' of all cooperative solution concepts. It has a very broad spectrum-it almost always exists, and it applies to political as well as economic and 'mixed' contexts. It very often gives results with significant conceptual content...which is often of independent interest, not connected with the idea of value in any obvious or transparent manner.... A very important point is that the value is mathematically tractable.
It lends itself to the application of mathematical methods from probability, measure theory, functional analysis and other areas. As a result, a very considerable body of theory has been built around the value; this theory may well be mathematically the richest and deepest in game theory. Of course this is intellectually pleasing, but that is not where its importance lies. Rather, its importance lies in the fact that this theory enables us to deal with the applications, to attack fairly complex models in a systematic manner and to solve them" (from Frontiers of Economics, edited by K. Arrow and S. Honkapohja, Basil Blackwell, Oxford, 1985).

These words were written [more than] thirty years ago. Since then, the theory and applications of the Shapley value have continued to flourish, and what was written then is all the more true today.

The work presaging market design and items 1-4 above are only some of the highlights of Shapley's contributions to coalitional game theory. There are many others.

We pass now to two of Shapley's most important contributions to the strategic ("noncooperative") theory.

5. Stochastic games, introduced by Shapley in the early 1950s to model the dynamics of ongoing game situations. Starting in the mid-1970s, this subject began to attract more and more attention from some of the top mathematical game theorists in the world, such as J.-F. Mertens, A. Neyman, E. Kohlberg, T. Bewley, and others. In the last twenty years, it has really taken off, with additional contributions by the above-named workers, in addition to extremely deep work by the French school, notably N. Vieille, J. Renault, and others, and by R. Simon. The mathematical depth and beauty of this work matches that of the work on the Shapley value (item 4 above), though it is of course something completely different. The theory also has important conceptual applications.

6. There are sometimes simple examples that are very important. One such example is the famous "Prisoner's dilemma", which has had a justifiably enormous impact on game theory and social science in general. Not as well known to the general public, but of equal importance, and well known to the cognoscenti, is "Shapley's game":

$\begin{array}{lll}0,0 & 4,5 & 5,4 \\ 5,4 & 0,0 & 4,5 \\ 4,5 & 5,4 & 0,0\end{array}$

This example has played a major role in the noncooperative theory. Originally advanced by Shapley to show that the important process known as "fictitious play" does not converge for nonzerosum games, it has since then taken on a life of its own; the first thing one does when working on a 


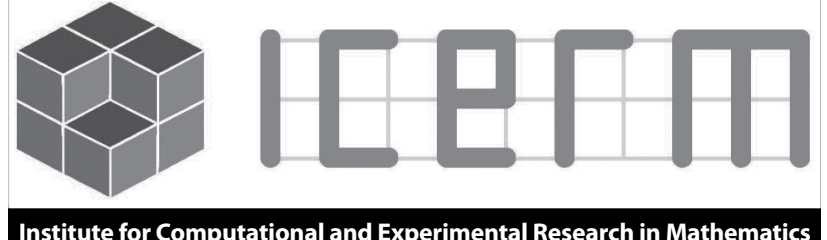

\section{Upcoming Programs and Events}

\section{SEMESTER-LONG PROGRAMS:}

(each with 3-4 associated workshops)

Low-dimensional Topology, Geometry, and Dynamics

Fall 2013: September 9 - December 6

Network Science and Graph Algorithms

Spring 2014: February 3 - May 9

\section{TOPICAL WORKSHOP:}

Issues in Solving the Boltzmann Equation for Aerospace Applications

June $3-7,2013$

\section{SUMMER PROGRAMS:}

Summer@ICERM (Undergraduate Summer Research) Geometry and Dynamics

June 17-August 9, 2013

IdeaLab (for Postdoctoral Researchers)

Topic 1: Tipping Points in Climate Systems

Topic 2: Towards Efficient Homomorphic Encryption

July 15-19, 2013

\section{SPECIAL EVENTS:}

Scratching the Surface in Dynamic Visual Effects Public lecture featuring Robert Bridson, UBC March 11, 2013

Research Experiences for Undergraduate Faculty (REUF) Co-sponsored with American Institute of Mathematics (AIM) July 22-26, 2013

Modern Mathematics Workshop (during SACNAS) October 3 - 6, 2013 in San Antonio, TX

Participation: ICERM welcomes applications for long- and short-term visitors. Support for local expenses may be provided. Decisions about online applications are typically made 1-3 months before a program, as space and funding permit. ICERM encourages women and members of underrepresented minorities to apply.

To learn more about ICERM's programs, organizers, confirmed program participants, and to submit an application, please visit our website:

\section{http://icerm.brown.edu}

About ICERM: The Institute for Computational and Experimental Research in Mathematics is a National Science Foundation Mathematics Institute at Brown University in Providence, RI. Its mission is to broaden the relationship between mathematics and computation. noncooperative theory is to see how it applies to Shapley's game.

The above represents only the tip of the iceberg. Shapley's work in game theory-both applied and mathematical-is truly astounding in scope, in depth, in beauty, and in importance. On each of these counts, Shapley has done more than all the previous game theory Nobelists, even when taken together.

\section{Alvin Roth: Biographical Sketch}

Alvin E. Roth was born in 1951 and received his Ph.D. in operations research from Stanford University in 1974. He has held positions at the University of Illinois (1974-1982) and the University of Pittsburgh (1982-1998). Since 1998 he has been George Gund Professor of Economics and Business Administration at Harvard University and the Harvard Business School. He is currently a visiting professor at Stanford University, where he will become a full faculty member in 2013. He has held visiting professorships at the Technion in Israel (1986), at the Hebrew University of Jerusalem (1995), and at the University of Tel Aviv (1995). He has been a research associate at the National Bureau of Economic Research since 1998. His honors include fellowships from the Guggenheim Foundation (1983-1984) and the Alfred P. Sloan Foundation (1984-1986); the Lanchester Prize of the Operations Research Society of America (1991); and (with Itai Ashlagi) the NKR Terasaki Medical Innovation Award of the American Transplant Congress (2012). He is also a fellow of the American Academy of Arts and Sciences.

\section{Lloyd Shapley: Biographical Sketch}

Lloyd S. Shapley was born in 1923 in Cambridge, Massachusetts. He received his Ph.D. from Princeton University in 1953. He has held positions at Princeton (1952-1954) and at the RAND Corporation (1948-1949, 1954-1981). He joined the faculty of the University of California, Los Angeles, in 1981 and is currently professor emeritus at UCLA. He has held visiting appointments at the California Institute of Technology (1955-1956), the Indian Statistical Institute (1978-1979), the Hebrew University of Jerusalem (1979-1980), and Catholic University of Louvain (1982). He was awarded the John von Neumann Theory Prize in 1981. He is a fellow of the Econometric Society, the American Academy of Arts and Sciences, and the National Academy of Sciences, and is a distinguished fellow of the American Economic Association.

-Elaine Kehoe 